\title{
On the Effect of Feedback Delay in the Downlink of Multiuser OFDM Systems
}

\author{
Mario Goldenbaum ${ }^{\star}$, Rudi Abi Akl ${ }^{\dagger}$, Stefan Valentin ${ }^{\ddagger}$ and Sławomir Stańczak ${ }^{\dagger \star}$ \\ * Heinrich-Hertz-Lehrstuhl für Informationstheorie und theoretische Informationstechnik, \\ Technische Universität Berlin, Einsteinufer 25, 10587 Berlin, Germany \\ ${ }^{\dagger}$ Fraunhofer Institute for Telecommunications Heinrich Hertz Institute, \\ Einsteinufer 37, 10587 Berlin, Germany \\ $\ddagger$ Bell Labs, Alcatel-Lucent, Lorenzstr. 10, 70435 Stuttgart, Germany
}

\begin{abstract}
Allocating wireless resources often relies on the accurate feedback of channel state information (CSI). In this paper, we study how strongly a multiuser OFDM downlink with single-cell scheduling and channel estimation suffers from feedback delay. Unlike previous work, we study this degradation for optimal joint power and rate allocation under fairness constraints. Comparing the performance of the ideal case to delayed CSI shows that (i) adjusting the scheduler's fairness cannot mitigate the strong performance loss due to feedback delay but that (ii) simple linear channel prediction is a powerful tool to do so.
\end{abstract}

Index Terms-Channel-aware scheduling, resource allocation, feedback delay, orthogonal frequency division multiplexing

\section{INTRODUCTION}

Channel-aware scheduling and resource allocation has become an integral component of many wireless communication systems as for example code division multiple access (CDMA), IEEE 802.16, and long term evolution (LTE). In these systems, adaptive modulation and coding adjusts the data rate of each user according to its channel state. Then, a central scheduler assigns the resources (i.e., power, frequency bands, antennas) to the users according to their rates, quality of service (QoS), and fairness requirements.

Such resource allocation provides tremendous multi-user diversity gains but requires the scheduler to accurately know the users' current channel state information (CSI) prior to the transmission. Extracting this information from the received signal is infeasible when fading and interference lead to different uplink and downlink channel states. With such nonreciprocal channels, we have to obtain the CSI from the users via feedback. Beside (i) errors due to measuring and quantizing CSI at the user side, the feedback adds (ii) transmission errors and (iii) feedback delay. Each of these three impairments causes a mismatch between the observed and the real CSI which can substantially decrease the scheduler's performance. Since feedback delay primarily defines the upper bound of the achievable data rate in many mobile scenarios [1], we focus only on this impairment for now.

The work of M. Goldenbaum was supported by the German Research Foundation (DFG) under grant STA 864/3-1 and the work of S. Stańczak by the German Ministry for Education and Research (BMBF) under grant $01 \mathrm{BU} 920$.

\section{A. Paper's Contributions}

In this paper we study the effect of feedback delay on gradient-based scheduling. Thereby, we make the following contributions:

- Unlike previous work [1], [2], we investigate the effect of feedback delay on the throughput-optimal joint power and subcarrier allocation as well as for general fairness constraints.

- We study maximum throughput and proportional fair scheduling as relevant special cases and show by simulation that both strategies suffer equally from outdated CSI. Consequently, one cannot compensate for feedback delay by adjusting the scheduler's fairness constraints.

- However, we show that outdated CSI is at least for a maximum throughput strategy still beneficial within a relevant delay regime. We demonstrate that simple linear prediction can mitigate the performance degradation due to feedback delay in a single step.

Although the effect of feedback delay and of linear prediction was studied in earlier work, this has neither been done for the optimal joint power and rate allocation nor for general utility-based fairness constraints (see Sec. I-C).

\section{B. Scenario}

We focus on a classic scenario in wireless cellular communication. During the downlink, a central scheduler allocates the wireless channel resources to the multiple users within a single cell. One scheduling approach to balance user-specific QoS and fairness requirements against a global and dynamic objective (i.e., maximizing the cell's sum rate) is called gradient-based scheduling [3].

Gradient-based algorithms select the transmission rate vector that maximizes the projection onto the gradient of the system's total utility [4]. The utility is a function of each user's mean throughput and quantifies fairness and other QoS considerations. In this paper, we apply gradient-based scheduling to an Orthogonal Frequency Division Multiplexing (OFDM) downlink which is the standard physical layer in modern communication systems (i.e., IEEE 802.11, IEEE 802.16, 3GPP LTE) [5]. In such an OFDM system, the resource allocation problem is solved by determining (i) a subset 
of users for transmission, (ii) the assignment of available subcarriers to selected users, and (iii) the transmission power for each subcarrier.

Following these steps, the gradient-based strategy maximizes the weighted sum throughput over the set of feasible rates once per time slot. Assuming efficient channel coding, it is feasible to model the rate per subcarrier by the mutual information of additive white Gaussian noise channels. By allowing users to time-share each subcarrier, we shall see that these assumptions lead to a tractable convex optimization problem.

\section{Related Work and Structure}

There is a large body of publications regarding optimal resource allocation in OFDM systems (see [7]-[8] just to name a few). However, most of these works are devoted to optimize static objective functions without fairness aspects and do not include the effect of feedback delay. Doing so, this paper follows the approach from [4] where the objective changes over time based on gradient-based scheduling.

Another closely related paper is [2] where the authors considered the impact of linear prediction on the throughput performance of the downlink OFDMA channel. The main difference to our work is that [2] assumed a heuristic (i.e., suboptimal) OFDMA scheduler, whereas in this work we consider an optimal scheduler over the joint space of power and subcarrier allocations. Moreover we study a class of utility maximization problems with throughput maximization and proportional fairness as special cases. Therefore, in contrast to [2], this paper shows the system performance in the presence of feedback delay and linear prediction under optimality conditions in the sense of utility maximization with perfect channel state information at the base station.

The rest of the paper is organized as follows. Sec. II introduces the system model while Sec. III describes the scheduling method for the optimal joint subcarrier and power allocation. Simulation results for the effect of feedback delay are investigated in Sec. IV. To mitigate this effect, we introduce a simple linear predictor and show the resulting improvement in Sec. V. ${ }^{1}$

\section{SYSTEM MODEL}

\section{A. OFDM Downlink Scenario}

Consider the downlink of a single cell in a cellular OFDM system consisting of a base station and a finite set $\mathcal{K}:=$ $\{1, \ldots,|\mathcal{K}|\}$ of arbitrarily numbered mobile users, as depicted in Fig.1. The system's bandwidth is divided into a finite set of flat subbands and we denote the corresponding finite set of subcarriers by $\mathcal{N}:=\{1, \ldots,|\mathcal{N}|\}$. Without loss of generality, we assume that each subband has a bandwidth equal to one

\footnotetext{
${ }^{1}$ Notation: $\mathbb{R}, \mathbb{R}_{+}, \mathbb{R}_{++}, \mathbb{C}, \mathbb{Z}$ denote the real, non-negative real, positive real, complex and integer numbers, respectively. Vectors are represented by bold lowercase letters and matrices by bold upper case letters. $\mathbf{1}_{n}$ denotes the vector of length $n$ of all ones, the proper complex normal distribution is described by $\mathcal{N}_{\mathbb{C}}(\cdot, \cdot)$ and $(\cdot)^{*}$ denotes the conjugate of a complex number. Expressions $\boldsymbol{A} \geq a$ or $\boldsymbol{a} \geq a$ with respect to a scalar $a$ are understood element-wise.
}

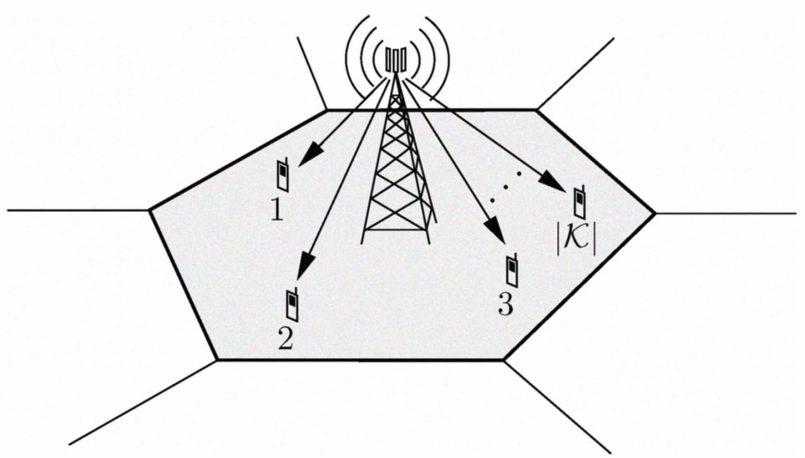

Fig. 1. Qualitative representation of the single-cell downlink scenario in a OFDM system consisting of a base station and a set $\mathcal{K}=\{1, \ldots,|\mathcal{K}|\}$ of arbitrarily numbered mobile users.

such that the system bandwidth is given by the number $|\mathcal{N}|$ of subcarriers as well.

At an arbitrary time instance $t \in \mathbb{Z}$,

$$
y_{k n}(t)=h_{k n}(t) s_{k n}(t)+n_{k n}(t)
$$

models the received complex baseband signal at mobile $k \in \mathcal{K}$ allocated to subcarrier $n \in \mathcal{N}$, where $h_{k n}(t) \in \mathbb{C}$ denotes the flat-fading channel gain between the base station and the mobile user at subcarrier $n \in \mathcal{N}$ (see Sec. II-B), $s_{k n}(t) \in \mathbb{C}$ is the corresponding transmit signal and $n_{k n}(t) \sim \mathcal{N}_{\mathbb{C}}\left(0, \sigma^{2}\right)$ the additive noise process with variance $\sigma^{2}>0$, for all $k \in \mathcal{K}$ and $n \in \mathcal{N}$. The average transmit energy per time slot of mobile $k$ allocated to subcarrier $n$ is denoted by $p_{k n}(t) \in \mathbb{R}_{+}$and we summarize all transmit energies into the matrix $\boldsymbol{P}(t):=$ $\left(p_{k n}(t)\right)_{k \in \mathcal{K}, n \in \mathcal{N}} \in \mathbb{R}_{+}^{|\mathcal{K}| \times|\mathcal{N}|}$.

The base station performs a scheduling method described in Sec. III consisting of a dynamic joint subcarrier and power allocation with the aim of allocating a certain rate vector $\boldsymbol{r}(t):=\left(r_{1}(t), \ldots, r_{|\mathcal{K}|}(t)\right) \in \mathbb{R}_{+}^{|\mathcal{K}|}$ with

$$
r_{k}(t)=\sum_{n \in \mathcal{N}} x_{k n}(t) \log \left(1+\frac{p_{k n}(t)\left|h_{k n}(t)\right|^{2}}{x_{k n}(t) \sigma^{2}}\right)
$$

the rate allocated to mobile $k \in \mathcal{K}$ at time $t \in \mathbb{Z}$. The coefficients $x_{k n}(t)$ describe the time sharing with respect to subcarrier $n \in \mathcal{N}$ between users (i.e., $x_{k n}(t)$ denotes the fraction of subcarrier $n$ to mobile $k$ at time instance $t){ }^{2}$ We collect them into the $|\mathcal{N}|$ vectors $\boldsymbol{x}_{n}(t):=\left(x_{1 n}, \ldots, x_{|\mathcal{K}| n}\right) \in \mathbb{R}_{+}^{|\mathcal{K}|}$.

Note that $\boldsymbol{r}(t)$ has to be an element of the feasible rate region $\mathcal{R}(t) \subseteq \mathbb{R}_{+}^{|\mathcal{K}|}$ defined as follows.

Definition 1 (Feasible Rate Region): Let $P \in \mathbb{R}_{+}$be the sum transmit power constraint at the base station. Then, for $t \in \mathbb{Z}$ and with (2) the feasible rate region is given by

$$
\begin{aligned}
\mathcal{R}(t):= & \left\{\boldsymbol{r} \in \mathbb{R}_{+}^{|\mathcal{K}|} \mid\left\|\boldsymbol{P}(t) \mathbf{1}_{|\mathcal{N}|}\right\|_{1} \leq P, \boldsymbol{P}(t) \geq 0,\right. \\
& \left.\forall n \in \mathcal{N}:\left\|\boldsymbol{x}_{n}(t)\right\|_{1} \leq 1, \boldsymbol{x}_{n}(t) \geq 0\right\} .
\end{aligned}
$$

Remark 1: It can be easily shown that the rate functions in (2) are concave with respect to the transmit energies $p_{k n}(t)$ and time-sharing coefficients $x_{k n}(t), k \in \mathcal{K}, n \in \mathcal{N}$.

\footnotetext{
${ }^{2}$ Note that $\forall x \in(-1, \infty): \lim _{x \rightarrow 0} x \log (1+1 / x)=0$.
} 


\section{B. Fading Model}

In this contribution, the deterministic complex envelope of the time varying flat channel gain between the base station and mobile $k \in \mathcal{K}$, allocated to subcarrier $n \in \mathcal{N}$, is modeled as [9]

$$
h_{k n}(t)=\sum_{\ell=1}^{L} a_{k n}^{(\ell)} \mathrm{e}^{i\left(2 \pi f_{k n}^{(\ell)} t+\phi_{k n}^{(\ell)}\right)}
$$

where $L \in \mathbb{N}$ determines the number of contributing paths and $a_{k n}^{(\ell)} \in \mathbb{R}, f_{k n}^{(\ell)} \in \mathbb{R}_{+}$and $\phi_{k n}^{(\ell)} \in[-\pi, \pi)$ are the corresponding attenuation, Doppler frequency and initial phase, respectively. If the mobile user is moving in a direction relative to the base station with corresponding speed $v_{k} \in \mathbb{R}_{+}$, the Doppler frequency can be written as $f_{k n}^{(\ell)}=f_{n} \frac{v_{k}}{c} \cos \left(\theta_{k \ell}\right)$, where $f_{n} \in$ $\mathbb{R}_{+}$denotes the $n^{\text {th }}$ subcarrier frequency, $c$ is the speed of light and $\theta_{k \ell} \in[-\pi, \pi)$ describes the angle between the $\ell^{\text {th }}$ incident wave and the movement direction of mobile $k \in \mathcal{K}^{3}{ }^{3}$ The set of maximum Doppler shifts is then given by $\left\{ \pm f_{k n}^{\max }=\right.$ $\left.\pm f_{n} \frac{v_{k}}{c}\right\}_{k \in \mathcal{K}, n \in \mathcal{N}}$ and will substantially affect the performance of the channel predictor in Sec. V.

\section{SCHEdULING Method}

Schedulers not only aim to maximize the cell's sum rate but have to account for fairness among the users. By optimizing the sum rate while addressing fairness by utility functions, channel-aware gradient-based scheduling [4] is one approach to do so. We will describe this approach as follows.

Let $\mathcal{T}(t):=\left\{t_{0}, \ldots, t-2, t-1\right\} \subset \mathbb{Z}$ be the time span up to $t-1 \in \mathbb{Z}$ since scheduler initialization at $t_{0} \in \mathbb{Z}$. Then, the average throughput of mobile user $k \in \mathcal{K}$ up to time $t \in \mathbb{Z}$ is defined as $\nu_{k}(t):=\frac{1}{|\mathcal{T}(t)|} \sum_{t^{\prime} \in \mathcal{T}(t)} r_{k}\left(t^{\prime}\right)$ and we summarize these rates in vector $\boldsymbol{\nu}(t):=\left(\nu_{1}(t), \ldots, \nu_{|\mathcal{K}|}(t)\right) \in \mathbb{R}_{+}^{|\mathcal{K}|}$. Based on $\boldsymbol{\nu}(t)$ we want to evaluate the system performance by a continuous concave (strictly increasing in each entry) utility function $U: \mathbb{R}_{+}^{|\mathcal{K}|} \rightarrow \mathbb{R}$. More specifically, we consider the space of utility functions of the kind $U(\boldsymbol{\nu}(t)):=$ $\sum_{k \in \mathcal{K}} U_{k}\left(\nu_{k}(t)\right), U_{k}: \mathbb{R}_{+} \rightarrow \mathbb{R}$, with

$$
U_{k}\left(\nu_{k}(t)\right)=\left\{\begin{array}{ll}
\frac{\nu_{k}(t)^{\alpha}}{\alpha}, & \alpha \in(0,1] \\
\log \left(\nu_{k}(t)\right), & \alpha=0
\end{array}, k \in \mathcal{K} .\right.
$$

The parameter $\alpha \in[0,1]$ allows us to adjust the degree of fairness between mobile users (see Sec. IV-B).

We are interested in a dynamic scheduling method that increases system performance by allocating at time $t \in \mathbb{Z}$ a rate vector $\boldsymbol{r}(t) \in \mathcal{R}(t)$ which maximizes the projection onto the gradient of $U(\boldsymbol{\nu}(t))$. The resulting scheduling problem can be explicitly stated as follows.

Problem 1: Let $t \in \mathbb{Z}$ be arbitrary but fixed and let $\left|h_{k n}(t)\right|^{2}$ and $\sigma^{2}$ be known to the base station for all $k \in \mathcal{K}$

\footnotetext{
${ }^{3}$ For Monte Carlo simulations in Sections IV and V we view (4) as the realization of a stochastic process.
}

and $n \in \mathcal{N}$. Then, the problem to solve is

$$
\max _{\boldsymbol{r}(t) \in \mathcal{R}(t)} \nabla_{\boldsymbol{\nu}} U(\boldsymbol{\nu}(t))^{T} \boldsymbol{r}(t)=\max _{\boldsymbol{r}(t) \in \mathcal{R}(t)} \sum_{k \in \mathcal{K}} \nu_{k}(t)^{\alpha-1} r_{k}(t)
$$

where $\nabla_{\boldsymbol{\nu}}$ denotes the Nabla operator with respect to $\boldsymbol{\nu}(t)$.

Remark 2: Because of Remark 1 and the convexity of the feasible rate region it follows that Problem 1 is convex and it can be shown that Slater's condition and strong duality holds.

For the rest of the paper we focus on the special case $x_{k n}(t) \in\{0,1\}$ for all $k \in \mathcal{K}, n \in \mathcal{N}$ and $t \in \mathbb{Z}$ (i.e., only one user per subcarrier is allowed).

The rate of mobile user $k \in \mathcal{K}$ at arbitrary $t \in \mathbb{Z}$, resulting from an optimal solution of Problem 1 (i.e., an optimal joint subcarrier and power allocation), is denoted as

$$
r_{k}^{\star}(t)=\sum_{n \in \mathcal{N}} x_{k n}^{\star}(t) \log \left(1+\frac{p_{k n}^{\star}(t)\left|h_{k n}(t)\right|^{2}}{x_{k n}^{\star}(t) \sigma^{2}}\right) .
$$

Provided that perfect CSI is available at the base station, (7) can be achieved by solving Problem 1 described as follows.

Using a standard Lagrange dual approach, the optimal dual variables can be obtained by solving (8) and (9) below via an iterative one dimensional search:

$$
\begin{gathered}
\lambda^{\star}=\arg \min _{\lambda \geq 0}\left\{\lambda P+\sum_{n \in \mathcal{N}} \mu_{n}^{\star}(\lambda)\right\} \\
\mu_{n}^{\star}(\lambda)=\max _{k \in \mathcal{K}}\left\{\mu_{k n}(\lambda)\right\}
\end{gathered}
$$

with

$$
\mu_{k n}(\lambda):=\nu_{k}(t)^{\alpha-1} f\left(\lambda, \nu_{k}(t)^{\alpha-1} \frac{\left|h_{k n}(t)\right|^{2}}{\sigma^{2}}\right)
$$

and $f: \mathbb{R}_{+} \times \mathbb{R}_{++} \rightarrow \mathbb{R}$,

$$
f(x, y)=\left\{\begin{array}{ll}
0, & x \geq y \\
\frac{x}{y}-\log \left(\frac{x}{y}\right)-1, & x<y
\end{array} .\right.
$$

The primal optimal subcarrier allocation for each $k \in \mathcal{K}$ and $n \in \mathcal{N}$ is then achieved by

$$
x_{k n}^{\star}(t)= \begin{cases}1, & k=\arg \max _{m \in \mathcal{K}}\left\{\mu_{m n}\left(\lambda^{\star}\right)\right\} \\ 0, & k^{\prime}=\arg \max _{m \in \mathcal{K}}\left\{\mu_{m n}\left(\lambda^{\star}\right)\right\}, k^{\prime} \neq k\end{cases}
$$

and the corresponding primal optimal power allocation by the water-filling solution

$$
p_{k n}^{\star}(t)=x_{k n}^{\star}(t) \frac{\sigma^{2}}{\left|h_{k n}(t)\right|^{2}} \max \left\{\frac{\nu_{k}(t)^{\alpha-1}\left|h_{k n}(t)\right|^{2}}{\lambda^{\star} \sigma^{2}}-1,0\right\} .
$$

Remark 3: Due to the constraints with respect to the subcarrier allocations in (3) it is possible that in the optimal subcarrier allocations (12) more than one mobile user is allocated to the same subcarrier. However, in such cases the mobile user with the largest transmit power is chosen which does not violates the sum power constraint $P$. 
TABLE I

DELAYS IN LTE THAT CONTRIBUTE TO THE OVERALL FEEDBACK DELAY $\tau$

\begin{tabular}{lll}
\hline Duration & Delay type & Node and cause of delay \\
\hline 1 & Process & User: Downlink channel estimation, encode \\
& Wait & Uplink subframe \\
1 & Transmit & User: Uplink data and CSI \\
1 & Process & BS: Uplink channel estimation, decode \\
$\geq 1$ & Process & BS: Resource allocation, encode \\
& Wait & Downlink subframe \\
1 & Transmit & BS: Downlink data and pilots \\
\hline
\end{tabular}

\section{EFFect of Feedback Delay}

The feedback delay $\tau$ forces the scheduler to use the outdated CSI $h_{k n}(t-\tau)$ where $t, \tau \in \mathbb{Z}$. When the downlink varies in time, this leads to a mismatch between (i) the actual channel state and (ii) the CSI used for scheduling, that is $h_{k n}(t-\tau) \neq h_{k n}(t)$. Based on (7), we denote the resulting rate allocation by

$$
r_{k}(t)=\sum_{n \in \mathcal{N}} x_{k n}^{\star}(t-\tau) \log \left(1+\frac{p_{k n}^{\star}(t-\tau)\left|h_{k n}(t)\right|^{2}}{x_{k n}^{\star}(t-\tau) \sigma^{2}}\right),
$$

$k \in \mathcal{K}$, and the solutions of Problem 1 by $p_{k n}^{\star}(t-\tau)$ and $x_{k n}^{\star}(t-\tau)$. Before we numerically compare the rate allocation under the influence of feedback delays (14) to the optimal allocation (7), let us briefly discuss the causes and typical values for the feedback delay.

\section{A. Feedback Delay}

By feedback delay $\tau$ we denote the total time lag between measuring the CSI and using it during a scheduled transmission. In most practical systems, $\tau$ consists of transmission time, processing time, and waiting time for a scheduling grant or for a different medium access control (MAC) scheme.

As an example, Tab. I summarizes such delays for LTE systems that follow current 3GPP standards [10]. These delays occur at the base station (BS) or at the user nodes, are given in subframes, and add up to $\tau$. The delay may vary between $5 \leq$ $\tau \leq 10$ subframes depending on how quickly the BS solves the resource allocation problem. Consequently, many current LTE systems have a feedback delay of at least 6 subframes.

\section{B. Maximum Throughput vs. Proportional Fair}

Working with component utility functions as in (5) allows us to adjust the fairness between maximum throughput and proportional fair [4], [11]. Maximum throughput achieves highest sum rate but discriminates users with low channel gain. A typical result are starving users at the cell edge. Proportional fair scheduling solves this issue at the cost of the maximum possible sum rate. Thus, both scheduling strategies are widely used in practice.

Now, we use the gradient-based scheduler described in Sec. III to study the effect of feedback delay on the average sum rate for both scheduling strategies. Setting $\alpha=1$ in (5) yields the maximum throughput strategy while we select proportional fairness by $\alpha=0$. As a third scheduling strategy we study "round robin", where each subcarrier is assigned to users in circular order determined by a deterministic function $g: \mathcal{K} \times$ $\mathcal{N} \times \mathbb{Z} \rightarrow\{0,1\}$, with equal power allocation over subcarriers and Gaussian codebooks. Since round robin does not depend on CSI feedback it provides a reasonable lower baseline for comparing the performance degradation due to outdated CSI. Hence, in comparison to (7) and (14) round robin leads to rate allocations

$$
r_{k}^{\mathrm{rr}}(t)=\sum_{n \in \mathcal{N}} g(k, n, t) \log \left(1+\frac{P\left|h_{k n}(t)\right|^{2}}{|\mathcal{N}| \sigma^{2}}\right),
$$

for each $k \in \mathcal{K}$ and $t \in \mathbb{Z}$.

Without loss of generality let $P$ and $\sigma^{2}$ such that $\frac{P}{\sqrt{\mathcal{N} \mid \sigma^{2}}}=1$. Then, the expected round robin signal-to-noise ratio (SNR) at user $k \in \mathcal{K}$ allocated to subcarrier $n \in \mathcal{N}$ is

$$
\overline{\operatorname{SNR}}_{k n}^{\mathrm{r}}(t):=\mathbb{E}\left\{\left|h_{k n}(t)\right|^{2}\right\}=\sum_{\ell=1}^{L} \mathbb{E}\left\{\left(a_{k n}^{(\ell)}\right)^{2}\right\}
$$

which we use to determine appropriate receiver operating points. $^{4}$

We simulate the downlink and feedback transmissions within a single cell using typical LTE parameters. We choose a subframe duration of $1 \mathrm{~ms}$ and fix the number of subcarriers to $|\mathcal{N}|=50$ [10]. For each subcarrier, we assume the channel to be frequency-flat (see Sec.II-B). Thus, $|\mathcal{N}|=50$ is equivalent to the 50 physical resource blocks (PRBs) of an LTE system with $10 \mathrm{MHz}$ bandwidth [5]. Finally, we assume $|\mathcal{K}|=10$ active users per cell, as common in macro cellular environments [12].

Each of the fading channels consist of $L=4$ i.i.d. propagation paths (cp. Sec. II-B). For the maximum Doppler shift, we choose $f_{k n}^{\max } \in\{10,44,66,100\} \mathrm{Hz}$, for all $k \in \mathcal{K}$ and $n \in \mathcal{N}$. These values correspond to maximum user speeds between 1.5 and $15 \mathrm{~m} / \mathrm{s}$ at $2 \mathrm{GHz}$ carrier frequency. Further, we introduce a certain degree of unfairness by setting the mean receiver operating points of two users $k_{1}, k_{2} \in \mathcal{K}$ to $\overline{\mathrm{SNR}}_{k_{1} n}^{\mathrm{T}}(t)=-10 \mathrm{~dB}$ and $\overline{\mathrm{SNR}}_{k_{1} n}^{\mathrm{Tr}}(t)=-20 \mathrm{~dB}$, respectively, for all $t \in \mathbb{Z}$ and all corresponding subcarriers (i.e., subcarriers $\left\{n \in \mathcal{N} \mid g\left(k_{1}, n, t\right)=1, t \in \mathbb{Z}\right\}$ and $\left\{n \in \mathcal{N} \mid g\left(k_{2}, n, t\right)=1, t \in \mathbb{Z}\right\}$ ) while the receiver operating points of the remaining mobile users $k \in \mathcal{K} \backslash\left\{k_{1}, k_{2}\right\}$ are set to $0 \mathrm{~dB}$. Fig. 2 shows that the maximum throughput strategy and the proportional fair rule suffer exactly the same from feedback delay. At this low Doppler shift, proportional fairness performs worse than round robin when the feedback delay exceeds $7.5 \mathrm{~ms}$. This means that even with slow channels, round robin should be preferred for higher feedback delays.

As feedback delay equally affects maximum throughput and proportional fair scheduling, we will limit to maximum throughput in the following section.

\footnotetext{
${ }^{4}$ Note that $\overline{\operatorname{SNR}}_{k^{\prime} n^{\prime}}^{\mathrm{rr}}\left(t^{\prime}\right) \neq 0$ only if $g\left(k^{\prime}, n^{\prime}, t^{\prime}\right)=1$ for $k^{\prime} \in \mathcal{K}, n^{\prime} \in \mathcal{N}$ and $t^{\prime} \in \mathbb{Z}$, respectively.
} 


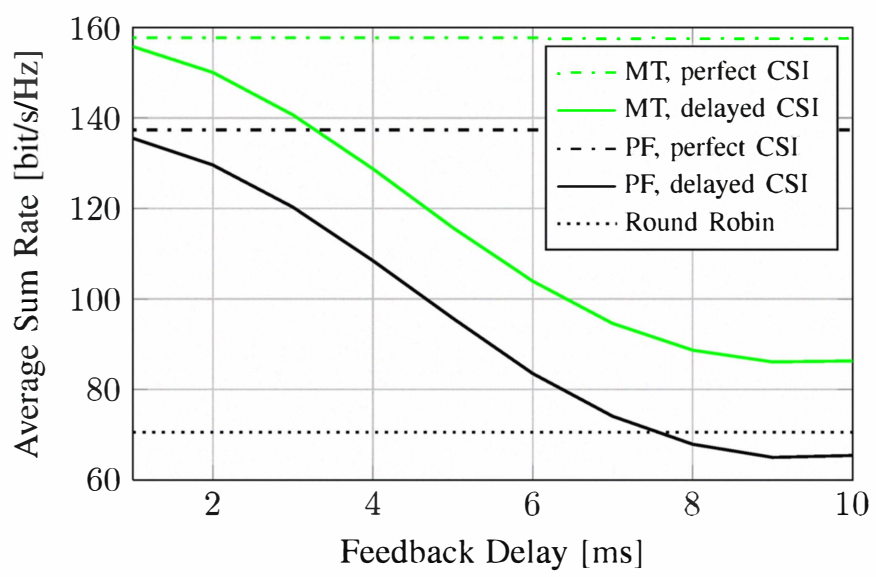

Fig. 2. Maximum Throughput (MT) ( $\alpha=1)$ vs. Proportional Fair (PF) $(\alpha=$ 0 ), averaged over $10 \cdot 10^{3}$ realizations, in a system with $|\mathcal{K}|=10$ mobile users and $|\mathcal{N}|=50$ subcarriers. The maximum Doppler shift is $f_{k n}^{\max }=44 \mathrm{~Hz}$, for all $k=1, \ldots, 10$ and $n=1, \ldots, 50$. The lower line corresponds to the CSI independent round robin strategy.

\section{Linear Channel Prediction}

To compensate for the degrading effect of feedback delay, a channel predictor can support the joint subcarrier and power allocation procedure by predicting future channel states from previously received CSI. To investigate the usefulness of prediction in the presence of optimal scheduling and a deterministic channel model, we will now apply a standard non-recursive prediction method from [13], [14] to scheduling and will provide some performance results.

\section{A. One-Step Prediction Method}

As in $(1), h_{k n}(t) \in \mathbb{C}$ represents the channel gain at an arbitrary but fixed time instance $t \in \mathbb{Z}$ between mobile $k \in \mathcal{K}$ allocated to subcarrier $n \in \mathcal{N}$, unknown to the base station. Using previously known samples of the channel, we define the prediction of $h_{k n}(t)$ as

$$
\hat{h}_{k n}(t):=-\sum_{\tau=1}^{p} \gamma_{k n}^{(\tau)} h_{k n}(t-\tau),
$$

with $\gamma_{k n}^{(\tau)} \in \mathbb{C}, \tau=1, \ldots, p$, the predictor coefficients and $p \in \mathbb{N}$ being the predictor depth (i.e., number of samples).

The question regarding an appropriate choice of the predictor coefficients can be answered by using the well known covariance method for deterministic time series [13], that is, solving the linear equation system

$$
-c_{k n}=C_{k n} \gamma_{k n}, k \in \mathcal{K}, n \in \mathcal{N},
$$

with respect to $\gamma_{k n}:=\left(\gamma_{k n}^{(1)}, \ldots, \gamma_{k n}^{(p)}\right) \in \mathbb{C}^{p}$, where $\boldsymbol{C}_{k n} \in$ $\mathbb{C}^{p \times p}$ represents a symmetric covariance matrix consisting of the elements $c_{n k}^{(\ell m)}:=\sum_{j=t_{0}}^{T} h_{k n}(j-\ell) h_{k n}^{*}(j-m)$, $1 \leq \ell, m \leq p$, and $\boldsymbol{c}_{k n} \in \mathbb{C}^{p}$ the column vector of elements $c_{k n}^{(0, m)}=\sum_{j=t_{0}}^{T} h_{k n}(j) h_{k n}^{*}(j-m), m=1, \ldots, p$. The integer values $t_{0}$ and $T, t_{0}<T<t$, define the observation interval which means that we are using the $|\mathcal{K}||\mathcal{N}|(p+T)$ previously received channel gains $\left\{h_{k n}\left(t_{0}-p\right), \ldots, h_{k n}(T)\right\}_{k \in \mathcal{K}, n \in \mathcal{N}}$ to

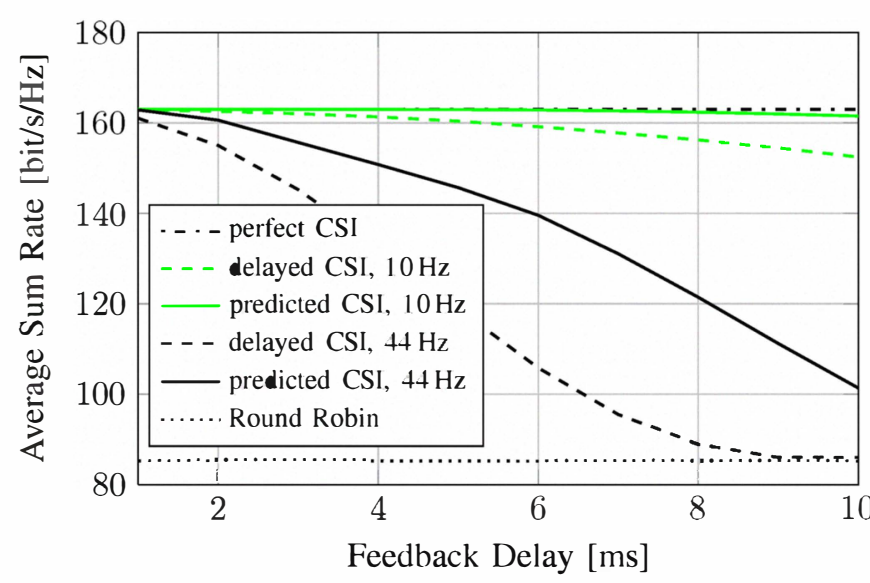

Fig. 3. Average sum rate (averaged over $10 \cdot 10^{3}$ realizations) as a function of feedback delay for a cell with $|\mathcal{K}|=10$ users, $|\mathcal{N}|=50$ subcarriers, a maximum Doppler shift of $f_{k n}^{\max }=f^{\max }=10 \mathrm{~Hz}$ and $44 \mathrm{~Hz}$, for all $k=1, \ldots, 10$ and $n=1, \ldots, 50$, and a prediction depth of $p=150$. The lower and upper lines correspond to Round Robin and perfect CSI at the base station.

identify $\left\{\gamma_{k n}\right\}_{k \in \mathcal{K}, n \in \mathcal{N}}$. Using the solution $\gamma_{k n}$ in (17) leads to the desired prediction $\hat{h}_{k n}(t)$.

Based on the predicted channel coefficients, solving Problem 1 with the scheduler described in Sec. III leads for arbitrary $t \in \mathbb{Z}$ to rate allocations

$$
r_{k}(t)=\sum_{n \in \mathcal{N}} \hat{x}_{k n}^{\star}(t) \log \left(1+\frac{\hat{p}_{k n}^{\star}(t)\left|h_{k n}(t)\right|^{2}}{\hat{x}_{k n}^{\star}(t) \sigma^{2}}\right),
$$

where $k \in \mathcal{K}$. To evaluate the suitability of a simple linear onestep predictor in practical situations, we will now compare the rate allocations from (19) to those from (14).

\section{B. Simulation Examples}

To evaluate how effective the above one-step prediction supports the scheduler, we choose a predictor depth of $p=150$ (see Sec. V-C for a motivation). Due to a channel sampling frequency of $10^{3} \mathrm{~Hz}, p$ can be considered to be in ms. The round robin operating point is now set to $0 \mathrm{~dB}$, for all $k \in \mathcal{K}$. All other simulation parameter match to Sec. IV-B.

The corresponding simulation results for different maximum Doppler shifts are depicted in Fig. 3 and Fig. 4. As one would expect, if the maximum Doppler shift is sufficiently low, the linear predictor performs well since it requires CSI observations to predict the future channel condition. When the channel evolves too quickly, the observed CSI may not contain sufficient information for prediction purposes and the predictor has difficulties to track the channel appropriately. As indicated in Fig. 4 it may even happen for high user mobility that scheduling with predicted CSI is worse than round robin.

\section{A Remark on the Choice of the Predictor Depth}

Finally, we study how the predictor depth $p \in \mathbb{N}$ affects the scheduling performance. Identifying the optimal value for $p$ is non-trivial since this is an estimation problem by itself [15]. Nonetheless, Monte Carlo simulation provides us a first look in Fig. 5. Here, the normalized average sum rate is shown 


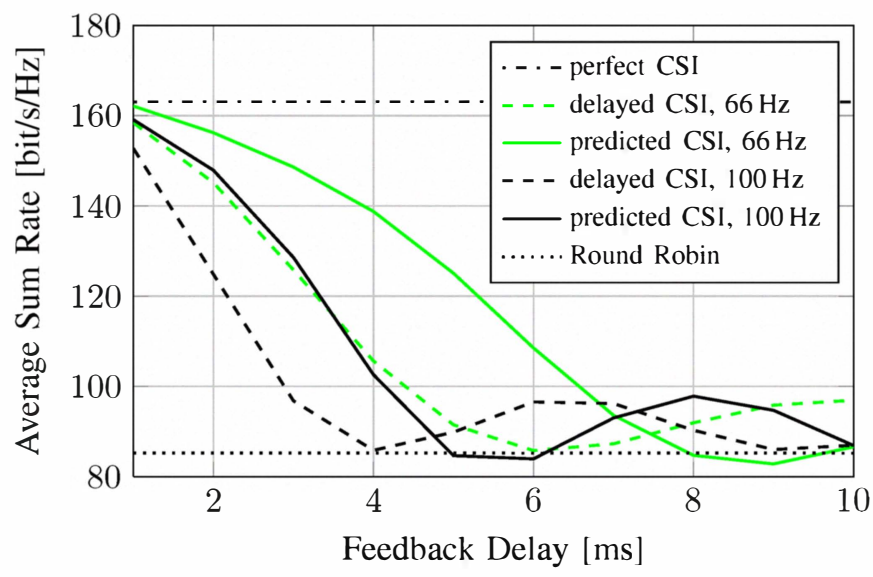

Fig. 4. Average sum rate (averaged over $10 \cdot 10^{3}$ realizations) as a function of feedback delay for a cell with $|\mathcal{K}|=10$ users, $|\mathcal{N}|=50$ subcarriers, a maximum Doppler shift of $f_{k n}^{\max }=f^{\max }=66 \mathrm{~Hz}$ and $100 \mathrm{~Hz}$, for all $k=1, \ldots, 10$ and $n=1, \ldots, 50$, and a prediction depth of $p=150$. The lower and upper lines correspond to round robin and perfect CSI at the base station.

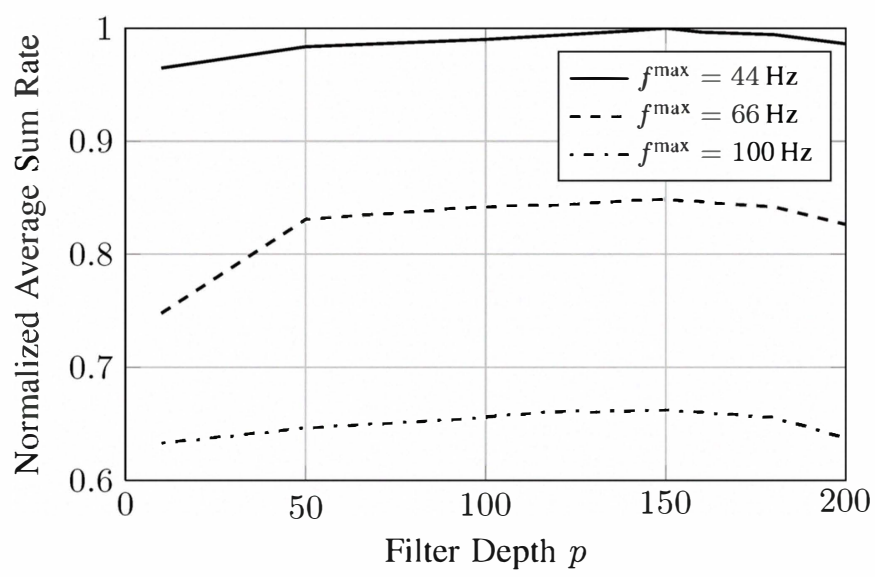

Fig. 5. Effect of the predictor depth $p$ on the average sum rate for a feedback delay of $\tau=5 \mathrm{~ms}$ and different maximum Doppler shifts $f^{\max } \in\{44,66,100\} \mathrm{Hz}$

versus $p$ for an exemplary fixed feedback delay $\tau=5 \mathrm{~ms}$ and different maximum Doppler shifts.

Apparently, there exists at least in the considered range of $p$ an unique global optimal predictor depth $p^{\star}=150$. This value is independent of the maximum Doppler shift and was used in the simulations of Sec. V-B. As shown, adjusting $p$ trades off the average sum rate versus computational complexity since choosing a higher $p$ increases processing delay.

\section{CONCLUSION}

We studied how strongly feedback delay degrades the performance of gradient-based scheduling. Accounting for different fairness constraints, round robin and linear prediction, we can conclude:

1) Maximum throughput and proportional fairness equally suffer from outdated CSI (see Fig. 2). Thus, increasing fairness does not change the effect of feedback delay.
2) Linear channel prediction substantially mitigates the effect of feedback delay. This simple but stable method already increased the rate by up to $30 \%$ (Fig. 3). This gain improves for slower channels where adaptive resource allocation (and thus CSI) becomes more relevant.

3) Outdated channel information is useful in relevant delay regions. Even at a typical LTE delay of $6 \mathrm{~ms}$, CSI based scheduling outperforms round robin by up to $27 \%$ without and by up to $65 \%$ with channel prediction (Fig. 3 and Fig. 4).

These conclusions give clear guidelines for designing future systems. To improve the sum rate, current feedback procedures need to be assisted by channel prediction. Our results for linear prediction show that this is feasible today. Furthermore, feedback delay needs to be reduced. This can be realized by introducing channel reciprocity [16] or by fast feedback protocols. Both are challenging fields for future research.

\section{ACKNOWLEDGEMENT}

The authors would like to thank Thorsten Wild (Bell Labs, Stuttgart) and Jonathan Ling (Bell Labs, Crawford Hill) for their helpful comments.

\section{REFERENCES}

[1] S. Valentin and T. Wild, "Studying the sum capacity of mobile multiuser diversity systems with feedback errors and delays," in Proc. IEEE 72nd Vehicular Technology Conference (VTC), Ottawa, Canada, Sep. 2010.

[2] Z. Shen, J. G. Andrews, and B. L. Evans, "Short range wireless channel prediction using local information," in Proc. 37th Asilomar Conference on Signals, Systems and Computers, Pacific Grove, USA, Nov. 2003, pp. $1147-1151$.

[3] V. G. Subramanian, R. A. Berry, and R. Agrawal, "Joint scheduling and resource allocation in cdma systems," IEEE Trans. Inf. Theory, vol. 56, no. 5, pp. 2416-2432, May 2010.

[4] J. Huang, V. G. Subramanian, R. Agrawal, and R. A. Berry, "Downlink scheduling and resource allocation for ofdm systems," IEEE Trans. Wireless Commun., vol. 8, no. 1, pp. 288-296, Jan. 2009.

[5] E. Dahlman, S. Parkvall, J. Skold, and P. Beming, $3 G$ Evolution: HSPA and LTE for Mobile Broadband. Academic Press, 2008.

[6] K. Seong, M. Mohseni, and J. M. Cioffi, "Optimal resource allocation for ofdma downlink systems," in Proc. IEEE International Symposium on Information Theory (ISIT), Seattle, USA, Jul. 2006, pp. 1394-1398.

[7] W. Yu and R. Lui, "Dual methods for nonconvex spectrum optimization of multicarrier systems," IEEE Trans. Commun., vol. 54, no. 7, pp. 1310 1322, Jul. 2006.

[8] G. Wunder and T. Michel, "Multiuser ofdma optimization: Algorithms and duality gap analysis," in Proc. International ITG Workshop on Smart Antennas (WSA), Darmstadt, Germany, Feb. 2008, pp. 233-244.

[9] S. Haykin and M. Moher, Modern Wireless Communications. New Jersey: Pearson Prentice Hall, 2005.

[10] 3GPP, "E-UTRA physical channels and modulation (release 9)," 3GPP, Technical Specification TS 36.211 V9.1.0, 2010.

[11] S. Stańczak, M. Wiczanowski, and H. Boche, Fundamentals of Resource Allocation in Wireless Networks. Berlin Heidelberg: Springer, 2009.

[12] 3GPP, "Further advancements for E-UTRA physical layer aspects (release 9)," 3GPP, Technical Report TR 36.814 V1.5.0, 2009.

[13] J. Makhoul, "Linear prediction: A tutorial review," Proc. IEEE, vol. 63, no. 4, pp. 561-580, Apr. 1975.

[14] T. Eyceoz, A. Duel-Hallen, and H. Hallen, "Deterministic channel modeling and long range prediction of fast fading mobile radio channels," IEEE Commun. Lett., vol. 2, no. 9, pp. 254-256, Sep. 1998.

[15] J. Rissanen, Stochastic Complexity in Statistical Inquiry. Singapore New Jersey: World Scientific Publishing, 1989.

[16] B. M. Hochwald and T. L. Marzetta, "Adapting a downlink array from uplink measurements," IEEE Trans. Signal Process., vol. 49, no. 3, pp. 642-653, Mar. 2001. 